\title{
L'expérience de l'industriel
}

\section{The experience of an industrial company}

\author{
par A. Adobes \\ Pour le Groupe Interactions Fluide-Structure de la Coordination \\ Thermohydraulique de la Division R\&D d'Electricité de France
}

The goal of the paper is to illustrate the experience of a company in the field of industry in solving mechanical problems related to the dynamics of a fluid coupled to a structure. We proceed by describing the strategies used by Electricite de France $(E D F)$ to face five problems related to the production of electricity : cracking of hydraulic turbines, improvement of piping systems performances, hydrodynamic instabilities of safety valves, tube damage in steam generators, and eventually the risk of a Loss Of Coolant Accident (LOCA).

\section{I — LES PROBLÈMES LIÉS AU COUPLAGE FLUIDE-STRUCTURE SONT FRÉQUENTS}

L'industriel doit parfois maîtriser une discipline scientifique sans lien direct apparent avec son métier de base. L'objectif de cet article est d'illustrer ce paradoxe à l'aide d'un exemple parmi d'autres : l'entreprise Electricité de France (EDF) développe des compétences dans le domaine du couplage fluide-structure, alors que son métier est la fourniture de courant électrique. Nous procéderons en nous appuyant sur cinq problèmes concrets d'origine vibratoire, qu'EDF ne pourrait surmonter sans considérer simultanément la structure et le fluide concernés : la fissuration de turbines hydrauliques, l'amélioration des performances des circuits de tuyauteries, les instabilités hydrodynamiques de soupapes de sûreté, l'endommagement des tubes de générateur de vapeur, enfin l'éventualité de la situation Accidentelle dite de Perte de Réfrigérant Primaire (APRP). Ces cinq problèmes et leurs solutions sont de natures différentes. Ils ne représentent pourtant que partiellement l'ensemble des problèmes vibratoires posés à EDF, et dont la résolution requiert des compétences en couplage fluide-structure. Sans dresser la liste exhaustive de ces problèmes, on peut en effet encore citer : l'usure des grappes de commande (qui permettent de piloter la réaction nucléaire en chaîne dans le cœur du réacteur), les vibrations des assemblages combustibles, ... Les Unités Opérationnelles $\mathrm{d}^{\prime} \mathrm{EDF}$ font donc face à des problèmes vibratoires multiples et multiformes qui partagent une composante fluide-structure. Pour les résoudre, ces Unités peuvent s'appuyer sur les com- pétences de la Division R\&D de l'entreprise. En complément de son organisation principale, cette Division s'est dotée de structures transversales pour favoriser les échanges sur des thèmes spécifiques. Ainsi, la récurrence des problèmes vibratoires liés au couplage fluide-structure a motivé la création du Groupe Interactions Fluide-Structure, auteur du présent article. Comme le montrent les paragraphes suivants, la panoplie des solutions apportées intègre : des essais sur site ou en laboratoire, le recours à la modélisation des phénomènes à l'aide de codes de calcul, enfin des procédures d'ajustement de modèle, permettant de s'assurer de la validité de tout futur calcul prédictif.

\section{II — L'EAU QUI FAIT TOURNER LES TURBINES PEUT AUSSI LES FAIRE FISSURER}

2.1 Quand une turbine entre en résonance sous l'ef-
fet de l'excitation fluide due aux jets d'eau

Chacun garde en mémoire l'effondrement du pont de Tacoma sur l'Hudson (USA) sous l'effet d'une excitation éolienne organisée. Plus généralement, l'action d'une force périodique peut faire entrer en résonance une structure et finir par mettre en péril son intégrité. Les turbines hydrauliques mises en service par EDF (figure 1) ne sont pas exemptes de ce danger potentiel. En effet, tournant à vitesse constante, elles sont mues par la pression qu'exercent périodiquement sur elles les jets d'eau disposés en couronne. 


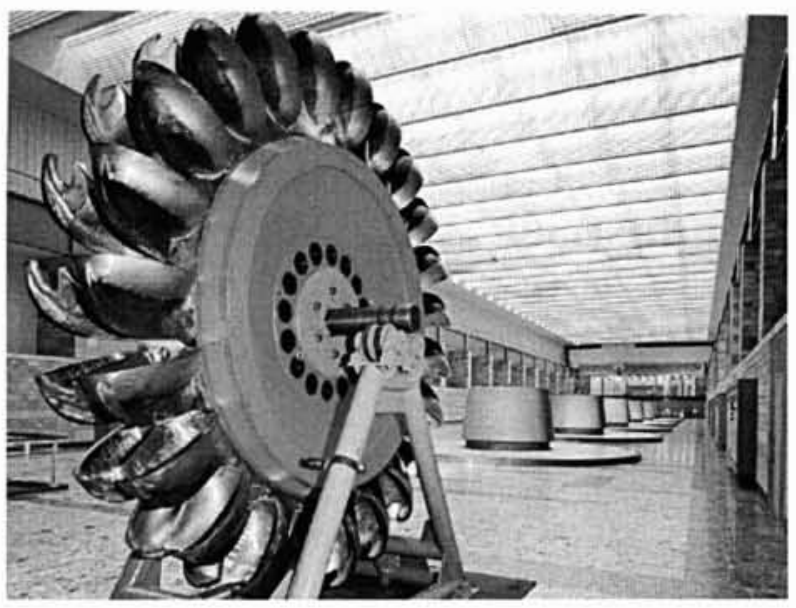

1. Turbine hydraulique PELTON à l'entreposage.

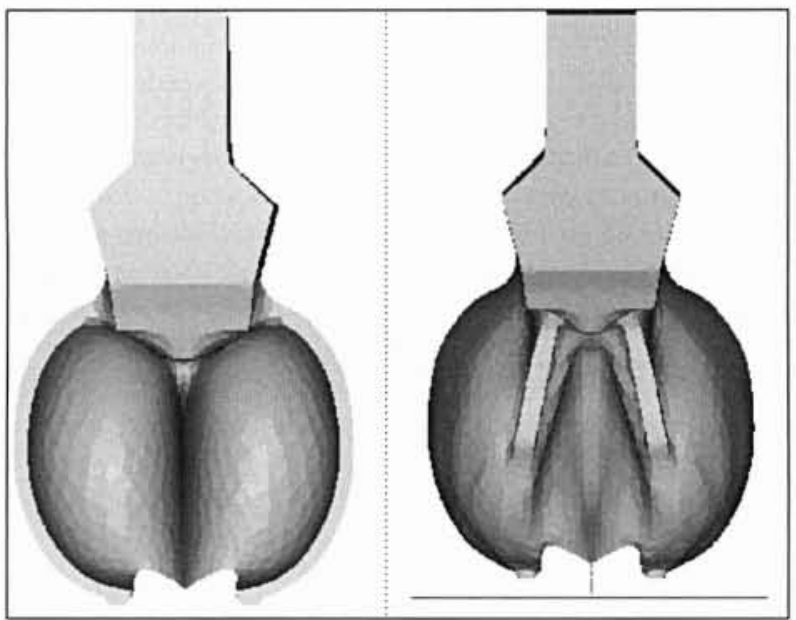

2. Intrados et extrados d'un modèle 3D de roue.

Pour éviter ce genre d'avatar, les constructeurs prennent des précautions lors du dimensionnement dynamique des roues. Cependant, ces dernières étant des structures riches en modes proches les uns des autres, les risques d'une coïncidence indésirable entre une composante fréquentielle de l'excitation périodique d'une part, et d'autre part la fréquence propre de l'un des modes de la structure, sont réels. Lorsque des vibrations excessives sont constatées sur une turbine, le cas mérite d'être instruit, non seulement pour éviter à court terme une rupture d'aube, coûteuse, voire dangereuse pour l'exploitant, mais aussi pour progresser encore en vue du dimensionnement des roues futures. Les roues de type PELTON mises en service successivement à la centrale hydraulique de la Combe d'Avrieux constituent à ce propos un cas d'école.

\subsection{Tirer parti d'un avatar pour progresser dans le dimensionnement dynamique}

Sur la première de ces roues, des contraintes doubles du niveau admissible sont détectées en service dès la mise en exploitation de la centrale. Les turbines suivantes, pourtant réalisées à l'identique, présentent des contraintes dynamiques acceptables. Il devient alors important de comprendre l'origine des contraintes excessives mesurées, ainsi que les raisons des écarts observés d'une roue à l'autre. Parmi les causes possibles de la fissuration, figure l'existence d'un niveau de contrainte dynamique excessif, qui serait lié à l'ac- crochage entre un harmonique de la fréquence fondamentale d'excitation et un mode propre de la roue en fonctionnement. La roue tournant à 500 tours/minute et le pic de contrainte se produisant à $600 \mathrm{Hertz}$, le 72 ème harmonique est soupçonné d'être à l'origine du problème. Afin d'analyser le problème, la Division R\&D d'EDF engage, de 1994 à 1998, un ensemble d'actions. Il est décidé de s'appuyer sur le cas concret de la roue qui a posé problème. L'objectif est de reproduire, à l'aide d'un modèle de comportement dynamique, le champ spatial de contrainte vibratoire anormalement élevée constaté en service. Si le modèle est satisfaisant, on aura prouvé une capacité à simuler le comportement dynamique d'une roue, voire à détecter de manière précoce une conception inadaptée, et, pourquoi pas, à proposer des modifications au stade de la planche à dessin.

\subsection{Construire pas à pas un modèle de comporte- ment dynamique de turbine PELTON}

Pour répondre aux besoins spécifiques d'EDF en modélisation mécanique ou thermique, la Division R\&D a développé dans les années 90 un code de calcul aux éléments

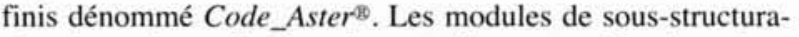
tion dynamique développés pour d'autres applications dans ce code ont été mis en œuvre pour concevoir un modèle dynamique de la première roue de la Combe d'Avrieux le plus représentatif possible. La saisie de la géométrie de la roue a été traitée avec soin en utilisant une technique de maillage de type "as built" (figure 2). Les fréquences et les déformées propres de la roue en air sous conditions d'appui libre ont été mesurées en laboratoire, puis simulées à l'aide de Code_Aster ${ }^{20}$. L'ajustement des valeurs des caractéristiques mécaniques de l'acier de la roue a permis d'atteindre une excellente concordance calculs/essais. Une étude paramétrique numérique a également été menée afin d'évaluer l'influence, sur le comportement modal de la roue, des conditions de fixation de cette dernière à l'arbre de l'alternateur. La modélisation de l'excitation appliquée par fluide sur la structure a été effectuée moyennant deux actions préliminaires. Tout d'abord, la masse ajoutée sur la roue par l'eau issue de la couronne de jets a été prise en compte en augmentant en conséquence la masse volumique de la roue lors des simulations. Cet ajustement a permis d'égaler la fréquence propre du mode à 9 diamètres nodaux avec la fréquence $(600 \mathrm{Hertz})$ du 72 ème harmonique de la vitesse de rotation. En effet, la topologie de la déformée modale de ce mode, appropriée avec l'excitation, en faisait le responsable le plus probable de la crise vibratoire observée. Ensuite, on a élaboré un modèle temporel de l'excitation dynamique de la roue par les jets d'eau. Bien que simplifié, ce modèle fournit un spectre de la force qui s'applique en chaque point d'impact des jets sur chaque auget.

\section{- 2.4 L'industriel a beaucoup appris de la modélisa- tion, y compris sur ses limites actuelles}

L'utilisation conjointe du modèle d'excitation fluide et du modèle de réponse dynamique de la roue a permis, dans un premier temps de valider l'hypothèse d'une "appropriation" de l'excitation de la roue avec un mode propre de flexion des augets à 9 diamètres nodaux et, dans un deuxième temps, de calculer les contraintes aux augets (figure 3 ). La valeur maximale des contraintes obtenue par le calcul de réponse (150 mégapascals) est cohérente avec les mesures en fonctionnement, ce qui valide la démarche de modélisation utili- 
sée. La modélisation numérique apparaît donc aujourd'hui comme étant un auxiliaire appréciable pour comprendre et reproduire les phénomènes de crises vibratoires qui peuvent surgir sur les turbines de type PELTON.

L'étude a aussi permis de cerner les limites actuelles de la modélisation. Il est ainsi apparu que les crises vibratoires ne peuvent pas encore être anticipées de manière fiable à la conception sur la seule base de calculs prédictifs. En effet, ces crises résultent d'une coïncidence très précise (à une fraction de pour cents près) entre une raie d'excitation et la fréquence d'un mode propre de la roue qui soit "appropriable". Or, pour l'instant, la méconnaissance relative des valeurs des différents paramètres d'entrée du calcul modal engendre une imprécision dans le calcul des fréquences propres de l'ordre de quelques pour cents, de sorte que l'on ne peut pas se passer d'un ajustement rigoureux du modèle dynamique de la roue, et donc de son analyse modale expérimentale préalable.

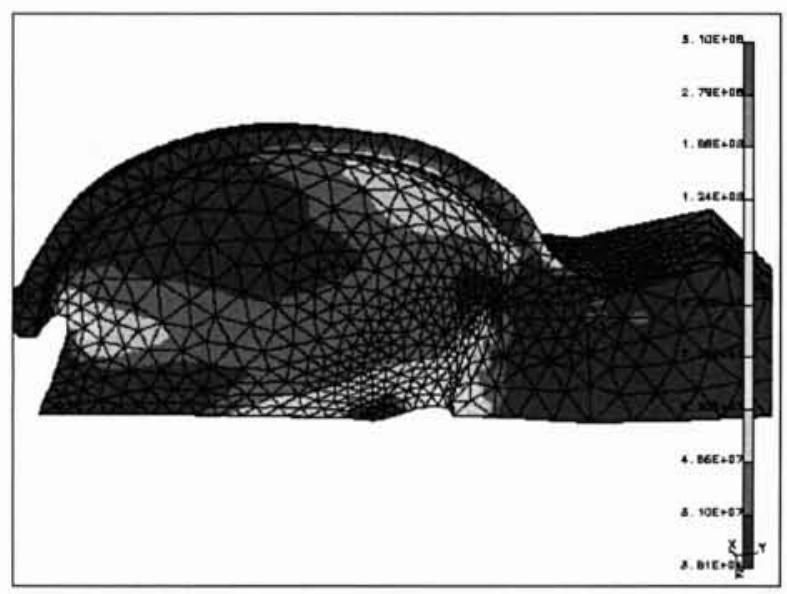

3. Contraintes sur l'intrados d'un $1 / 2$ auget à $600 \mathrm{~Hz}$.

\section{III $\square$ MISES EN VIBRATION PAR LES FLUIDES QU'ELLES VÉHICU- LENT, CERTAINES TUYAUTERIES PEUVENT CÉDER}

\subsection{L'intégrité des circuits de tuyauteries impor- tants pour la sûreté doit être préservée}

Les matériels présents sur les réseaux de tuyauteries ont pour rôle d'assurer un niveau de pression statique et de débit nécessaire aux différentes conditions d'exploitation des centrales nucléaires. Ces grandeurs thermohydrauliques stationnaires s'accompagnent de fluctuations instationnaires de pression et de débit, engendrées par les matériels. La nature acoustique de ces dernières entraîne la propagation de ces phénomènes sur de longues distances et la mise en vibration des lignes de tuyauteries. Les circuits Importants Pour la Sûreté (IPS) sont conçus et exploités pour assurer une fonction donnée. Leur intégrité doit donc être préservée. Dès le début des années 1980, EDF a donc mis l'accent, à la fois sur la nécessité d'améliorer la prise en compte à la conception des phénomènes vibratoires sur les lignes principales et les piquages, et sur l'intérêt de vérifier le bon comportement vibratoire des points singuliers, zones potentielles de vibration, dans des conditions de fonctionnement pénalisantes visà-vis de ce phénomène. Du point de vue vibratoire, l'enjeu était de fournir un résultat exploitable, c'est-à-dire, non pas en termes de modes et d'amortissement, mais, comme dans le cas des roues PELTON, en termes de contrainte à comparer à une limite d'endurance. Pour y parvenir, la Division R\&D a choisi de capitaliser le savoir-faire existant dans le code CIRCUS.

\section{- 3.2 Un outil-métier a été développé pour capitaliser le savoir-faire en dynamique des circuits}

CIRCUS est un outil logiciel qui permet d'étudier le comportement hydraulique, acoustique, statique, sismique et vibratoire des réseaux de tuyauteries soumis à des excitations induites par un écoulement, c'est-à-dire dans les conditions du fonctionnement. Il permet en particulier de déterminer les phénomènes acoustiques ou vibratoires susceptibles de se propager, de les localiser, de les quantifier, enfin d'évaluer les risques d'endommagement, et de faire évoluer le comportement du circuit en modifiant les paramètres de dimensionnement ou de fonctionnement. Dans ce dernier cas, CIRCUS permet également de vérifier que les modifications proposées conduisent à des situations compatibles avec les aspects de conception réglementaire d'usage dans le domaine nucléaire.

CIRCUS dispose d'une base de données de singularités acoustiques passives (vannes, diaphragmes, changements de section, ...) ou actives (pompes, ...) pour reconstituer les sources d'excitation. Cette base rassemble, sous un formalisme unique, d'une part des formulations analytiques adimensionnalisées vis-à-vis de la géométrie et des conditions de fonctionnement et, d'autre part, des résultats d'essais relatifs à des matériels particuliers du parc nucléaire français obtenus en fonctionnement sur site ou sur boucles d'essais. Les sources obtenues sur site peuvent être analysées avec le code, avant d'être archivées dans la base de données, ce qui assure le retour d'expérience des connaissances acquises sur les mécanismes de génération et de propagation du bruit et des vibrations dans les circuits en exploitation. Elles sont alors mises à la disposition de l'utilisateur pour les besoins de ses études, de façon fiable et transparente. Il est ainsi possible d'utiliser des sollicitations dynamiques réelles et des conditions limites, via la prise en compte de mesures issues d'essais.

Le réseau de tuyauteries est modélisé par la théorie des ondes planes et des poutres. La méthode de calcul est basée sur une décomposition élémentaire mixte de matrices de transfert/éléments finis, connue sous le nom de matrices de rigidité dynamique exacte. Ces éléments prennent en compte, s'il y a lieu, le couplage fluide-structure (coude, piquage, changement de section, ...). La résolution du problème qui nécessite un nombre d'éléments réduits par rapport à une méthode d'éléments finis s'opère par assemblage puis résolution d'un système respectant les équations d'équilibre aux jonctions entre les éléments.

Après cette brève présentation du logiciel CIRCUS, le paragraphe ci-après vise à en illustrer l'intérêt pour EDF à l'aide d'un exemple d'application.

\section{- 3.3 De l'intérêt de la modélisation pour réduire des contraintes dynamiques excessives}

Les études du circuit d'Injection de Sécurité Moyenne Pression des paliers nucléaires 1300 et 1400 mégawatts font suite au constat de mouvement de collecteur (déplacements de valeurs élevées) sur la ligne de tuyauteries qui relie la pompe de gavage à la pompe principale du circuit. Ces 
vibrations sont dues aux sources engendrées par le fonctionnement à débit partiel de certaines pompes et sont des sources potentielles d'endommagement par fatigue vibratoire. La configuration est modélisée à l'aide du code CIRCUS. Le modèle mis en oeuvre prend en compte, à la fois les sources acoustiques des pompes, et les effets d'interaction fluidestructure. Les sources d'excitation dues aux pompes n'ayant pas été caractérisées expérimentalement, c'est le modèle prévisionnel de comportement dynamique de la pompe disponible dans la base de données du code qui est utilisé.

D'un point de vue strictement mécanique (c'est-à-dire en négligeant les effets de couplage liés à la présence du fluide), seule serait à modéliser la portion de circuit comprise entre les deux pompes. En effet, on peut considérer les brides de raccordement de ces pompes comme étant des points fixes et, par conséquent, que le collecteur est mécaniquement découplé du reste du circuit. Cette approche n'est cependant plus valable dès lors que l'on prend en considération le couplage fluide-structure. En effet, les conditions aux limites acoustiques au niveau des brides sont inconnues. Comme les ondes acoustiques se propagent au-delà des pompes dans tous les éléments de tuyauteries en connexion directe avec la ligne, il devient nécessaire d'inclure dans la modélisation les circuits amont et aval des pompes, remontant ainsi jusqu'aux organes d'isolement.

Pour l'étude menée sur le palier 1300 mégawatts, la modélisation acoustique des tronçons amont et aval a été construite avec les conditions aux limites de la propagation acoustique, conformes aux régimes de fonctionnement observés lors d'essais sur site. Afin de diminuer les niveaux vibratoires mesurés, il a été proposé de modifier le supportage du circuit inter-pompes du palier 1300 mégawatts par l'adjonction d'une butée dans les directions latérale $\mathrm{X}$ et $\mathrm{Y}$ (figures 4 et 5 ).

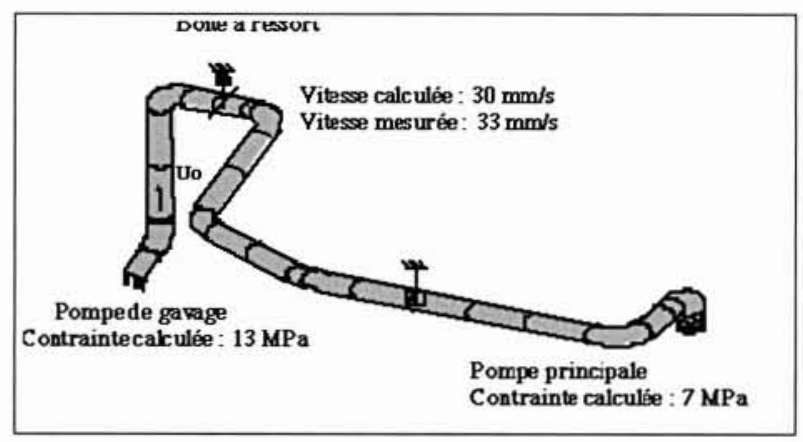

4. Contrainte de Tresca maximale sur la bride de raccordement d'une pompe de gavage avant modification.

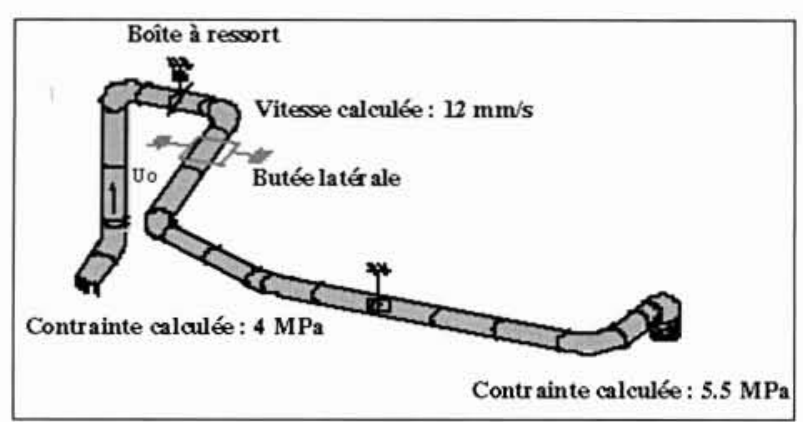

5. Contrainte de Tresca maximale après la modification simulée avec CIRCUS.
La modification intervient à l'endroit où la vitesse vibratoire de la ligne est maximale. La simulation de la modification à l'aide de CIRCUS met en évidence la forte diminution des vitesses vibratoires ainsi qu'une diminution d'un facteur 3 de la contrainte dynamique maximale au niveau de la bride de raccordement de la pompe de gavage. On observe également une amélioration de la répartition des niveaux vibratoires le long de la portion de circuit comprise entre les deux pompes. La zone la plus sollicitée du tronçon modifié est observée maintenant au niveau de la bride de raccordement de la pompe principale, le niveau de contrainte demeurant néanmoins admissible. Par ailleurs, il a été vérifié que la modification des fréquences propres du tronçon n'engendre pas de nouveau phénomène de couplage.

En conclusion, si la modification proposée s'impose d'ellemême pour un "tuyauteur", le fait d'avoir pu quantifier, d'une part les niveaux vibratoires globaux, et d'autre part le nouveau contenu fréquentiel du circuit, avant de réaliser la modification, a permis d'améliorer le comportement dynamique du circuit plus sûrement, tout en minimisant les risques et les coûts induits.

\section{IV — LES INSTABILITÉS HYDRODYNA- MIQUES DANS LES SOUPAPES DE SÛRETÉ}

\subsection{Quand le couplage fluide-structure se manifeste au niveau du clapet d'une soupape}

On vient de voir que la tenue mécanique des circuits de tuyauteries dans leur ensemble est analysée à l'aide de l'outil-métier CIRCUS. Cependant, certains composants de ces circuits, tels que les soupapes de sûreté des centrales nucléaires, requièrent à eux seuls une analyse détaillée, nécessitant elle aussi une bonne compréhension des phénomènes de couplage fluide-structure. Le phénomène préoccupant est en l'occurrence celui des battements de clapet engendrés par les fluctuations de pression qui se produisent lors des opérations d'ouverture ou de fermeture. Ces battements seraient en effet préjudiciables au bon fonctionnement d'une centrale ; ils pourraient provoquer un endommagement mécanique, lui-même susceptible d'engendrer des pertes d'étanchéité et de perturber le débit dans le circuit. La Division R\&D d'EDF conduit donc depuis plusieurs années des études numériques et expérimentales visant à mettre au point une modélisation des phénomènes observés qui permette d'étudier le comportement hydrodynamique des soupapes. Le but de cette modélisation est double : d'une part être en mesure de conduire, par simulation numérique, des études de mécanique des fluides pour répondre aux questions soulevées par l'Autorité de Sûreté et, d'autre part, réduire le nombre d'essais à effectuer sur boucle lors de la construction des futurs paliers nucléaires. Le développement de cette démarche de modélisation suit trois axes principaux. Deux de ces axes sont numériques, le troisième est expérimental.

\section{- 4.2 La démarche de modélisation numérique s'adapte aux butées techniques actuelles}

Les battements du clapet sont engendrés par une résonance hydroacoustique qui s'établit le long de la ligne d'alimentation de la soupape. Ce type de résonance est largement gouverné par la longueur de la ligne. Une approche monodimensionnelle s'avère donc être bien adaptée à la modélisation du phénomène. Par ailleurs, l'ouverture de la soupape 
s'accompagne d'une variation brutale de la pression, qui provoque à son tour une vaporisation partielle de l'écoulement liquide. Seul le choix d'une modélisation monodimensionnelle permet actuellement de prendre en compte le facteur de complexité lié à la nature diphasique de l'écoulement, surtout lorsque ce dernier est instationnaire. En effet, une approche plus fine $2 \mathrm{D}$ ou $3 \mathrm{D}$ de ce type de configuration est encore trop coûteuse en temps de calcul.

Cependant, pour mieux connaitre la nature des écoulements à l'intérieur de la soupape, une modélisation bidimensionnelle, puis tridimensionnelle a été conduite à l'aide des codes de calcul de mécanique des fluides N3S-EF et N3S-NATUR dans le cas d'écoulements monophasiques. Les cas d'écoulements incompressibles ont ainsi été traités. Dans un premier temps, les écoulements incompressibles observés dans une maquette de soupape en plexiglas ont été reproduits à l'aide de N3S-EF (en géométrie fixe). Pour intégrer la dynamique du clapet dans la modélisation de l'écoulement à l'intérieur de la soupape, le modèle a été enrichi par intégration de la méthode ALE (Arbitrary Lagrangian Eulerian) qui permet de calculer les écoulements à l'intérieur d'un volume déformable. Il est alors possible de prendre en compte le couplage entre l'écoulement d'eau (le fluide) et le clapet (la structure).

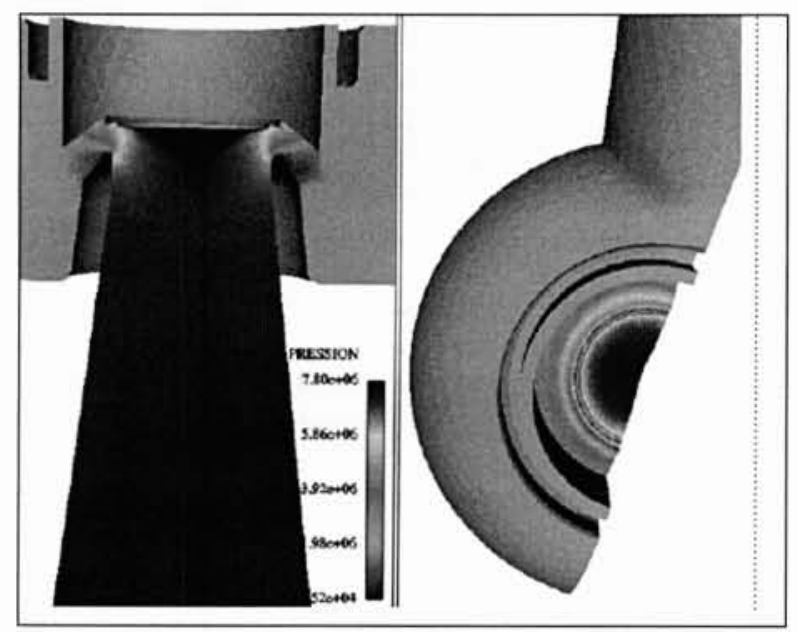

6. Champ de pression calculé dans un écoulement de vapeur dans une soupape de générateur de vapeur

La modélisation permet de simuler le mouvement de la paroi du clapet qui se déplace sous l'action de la pression appliquée, avec modification en retour du champ de pression dans le fluide. Cette approche numérique a été mise au point dans l'environnement du code N3S-EF. Elle a ensuite été testée sur le code N3S-NATUR, pour des écoulements compressibles, sur modèle $2 \mathrm{D}$ axisymétrique. Par ailleurs, sur un modèle $3 \mathrm{D}$, la comparaison avec l'expérience a montré que l'on arrive à approcher le débit vapeur d'une soupape avec une précision de $8 \%$ environ. La figure 6 permet d'apprécier la détente de la vapeur à la sortie de la soupape.

\subsection{Les calculs sont validés à l'aide d'essais effec- tués sur des soupapes réelles}

La mise au point de toute modélisation requiert la confrontation avec l'expérience. Pour cela, une soupape de générateur de vapeur du palier nucléaire 900 mégawatts de type Bopp \& Reuther a été équipée de capteurs de pression et de thermocouples, de manière à constituer une base de données thermodynamiques exploitable dans les années à venir. Cette base de données est alimentée par des essais en eau et en vapeur. D'autres essais sont prévus ultérieurement pour caractériser la physique du changement de phase et appuyer le développement du modèle diphasique.

\section{V — L'ENDOMMAGEMENT D'ORIGINE VIBRATOIRE DES TUBES DE GV}

\subsection{Dans les GV, il faut éviter qu'un tube n'entre en instabilité sous l'action de l'écoulement}

Le faisceau de tubes d'un générateur de vapeur de réacteur à eau pressurisée est soumis, en fonctionnement, à des écoulements parallèles aux tubes en partie droite et transverses aux tubes en partie cintrée ainsi que dans la zone d'entrée du faisceau (figure 7). Les différentes études de R\&D engagées dans le monde depuis le début des années 1970, ainsi que le retour d'expérience des générateurs de vapeur $(\mathrm{GV})$ en exploitation, ont montré que les faisceaux excités par un écoulement transverse peuvent présenter des risques d'origine vibratoire. Ces derniers sont susceptibles d'engendrer un endommagement prématuré des tubes, par exemple par usure au niveau des supports, ou par fatigue vibratoire. D'un point de vue industriel, il est donc important de modéliser finement l'ensemble des phénomènes à l'origine de ces endommagements, afin d'optimiser la politique de maintenance des appareils en fonctionnement, de définir les actions correctives à entreprendre sur ces matériels, et d'améliorer la conception des matériels futurs ou de remplacement.

\subsection{Savoir calculer l'usure ou la fatigue vibratoire éventuelles d'un tube}

Pour répondre à ces besoins, comme dans le cas de l'analyse des vibrations des circuits de tuyauteries, un logiciel spécifique a été développé par la Division R\&D. Cet outil-métier, dénommé GEVIBUS, permet la réalisation d'un calcul complet de mécanique d'un tube de GV excité par un écoulement, allant de l'estimation des paramètres thermohydrauliques jusqu'au calcul de l'usure ou de la fatigue vibratoire éventuelles.

GEVIBUS a été réalisé autour du code THYC pour la caractérisation de l'écoulement et, une nouvelle fois, autour de Code_Aster ${ }^{30}$, employé en l'occurrence pour prendre en compte, outre les aspects dynamique des structures et couplage fluide-structure, l'endommagement par usure ou fatigue. L'outil-métier a été conçu avant tout pour être un outil industriel, c'est-à-dire simple d'utilisation et ne nécessitant qu'une connaissance "industrielle" du composant pour mener à bien un calcul. Une Interface Homme Machine spécifique a donc été développée pour faciliter l'entrée des données de calcul, traiter ces données pour leur utilisation par le code THYC et Code_Aster "is , puis gérer l'ensemble des calculs de dynamique et d'endommagement réalisés par Code_Aster 3 . Un calcul industriel complet se subdivise en cinq étapes principales :

1 - Calcul thermohydraulique du GV.

2 - Prise en compte des phénomènes d'interaction fluidestructure dans le faisceau de tubes.

3 - Calcul dynamique linéaire ou non linéaire de la réponse de la structure.

4 - Estimation de l'usure.

5 - Estimation de la fatigue vibratoire.

Les quatre dernières étapes du calcul sont assurées grâce aux fonctionnalités développées dans Code_Aster ${ }^{\text {BB }}$.

Mis en exploitation en 1996, l'outil-métier GEVIBUS est devenu un outil appréciable pour le calcul de l'endommage- 


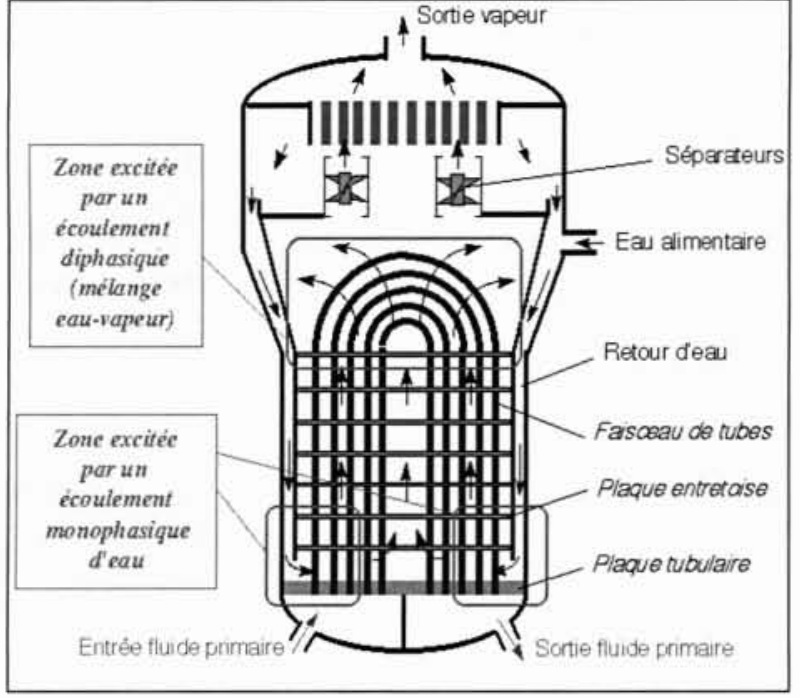

7. Schéma de principe d'un générateur de vapeur.

ment par usure ou fatigue vibratoire. Il est utilisé par EDF dans la constitution de dossiers pour les Autorités de Sûreté ou le contrôle de notes de calcul émises par le constructeur FRAMATOME.

\subsection{Certaines études contribuent à une meilleure optimisation du programme de maintenance}

Récemment, un problème industriel important a été traité à l'aide de GEVIBUS : le phénomène de dégradations de plaques entretoises dans les générateurs de vapeur. Ce phénomène, constaté sur certaines tranches du parc nucléaire français, se traduit par un défaut local de maintien de certains tubes au niveau de leurs supports, dans des zones pouvant se révéler sensibles vis-à-vis des phénomènes de couplage fluide-structure. Différentes configurations dégradées ont été étudiées avec GEVIBUS et ont permis de déterminer avec précision les tubes pouvant présenter des risques d'endommagement, en fonction de leur "environnement" (figure 8). Ce que l'on appelle ici "environnement" est essen- tiellement le nombre de tubes, voisins du tube considéré, affectés par une dégradation de la plaque. Ce paramètre "environnement", déterminant vis-à-vis des phénomènes d'interaction fluide-structure, a été le facteur principal permettant de statuer sur les risques d'instabilité des tubes. Les résultats de l'ensemble de ces calculs ont ensuite été exploités afin de définir et d'optimiser un programme de maintenance adapté aux différents générateurs de vapeur concernés. Les études correspondantes ont ainsi permis d'obtenir des gains qui peuvent être chiffrés en termes de coût d'intervention, en termes de pourcentage de tubes bouchés dans les GV (et donc maintien des conditions de fonctionnement nominales), mais aussi en termes de débits de doses reçus par le personnel assurant la maintenance.

\section{VI $\square$ LA PRISE EN COMPTE DE L'ÉVEN- TUALITÉ D'UN APRP}

Les composants du Circuit Primaire Principal (CPP) des centrales nucléaires à eau pressurisée (figure 9) sont dimensionnés pour pouvoir supporter un ensemble de situations accidentelles hypothétiques, dont l'Accident de Perte de Réfrigérant Primaire (APRP).

L'APRP correspond à la dépressurisation rapide d'une tuyauterie du CPP par suite de sa rupture brusque et totale. Au cours de cette dépressurisation, apparaissent les effets de couplage fluide-structure entre l'eau primaire et les structures du CPP. Ces effets sont dus, d'une part au déséquilibre des forces thermohydrauliques (décompression et vaporisation rapide du fluide), et d'autre part à des sollicitations purement mécaniques, liées à la rupture de la tuyauterie et au déplacement induit par la perte de l'effet de fond. Pendant quelques centaines de millisecondes après la rupture, les tuyauteries et les structures internes du réacteur sont alors fortement sollicitées.

Le maintien des fonctions importantes de sûreté pendant et/ou à la suite d'une situation accidentelle telle que l'accident de perte de réfrigérant primaire est démontré en menant des études sur sa simulation numérique dans le cadre des dossiers de dimensionnement. Mais, au-delà des situations accidentelles de référence, ou en cas de prolongement de la durée de vie d'une centrale, EDF doit montrer que ses centrales présentent des marges de sûreté.

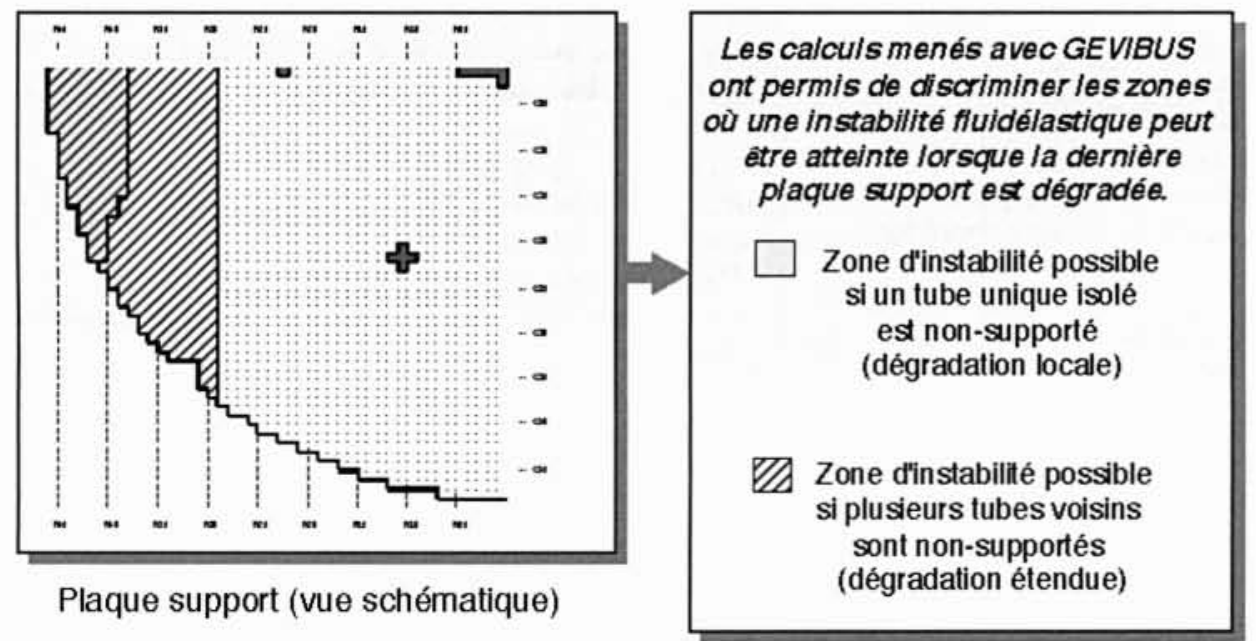

8. Application industrielle de GEVIBUS : les dégradations de plaques entretoises. 


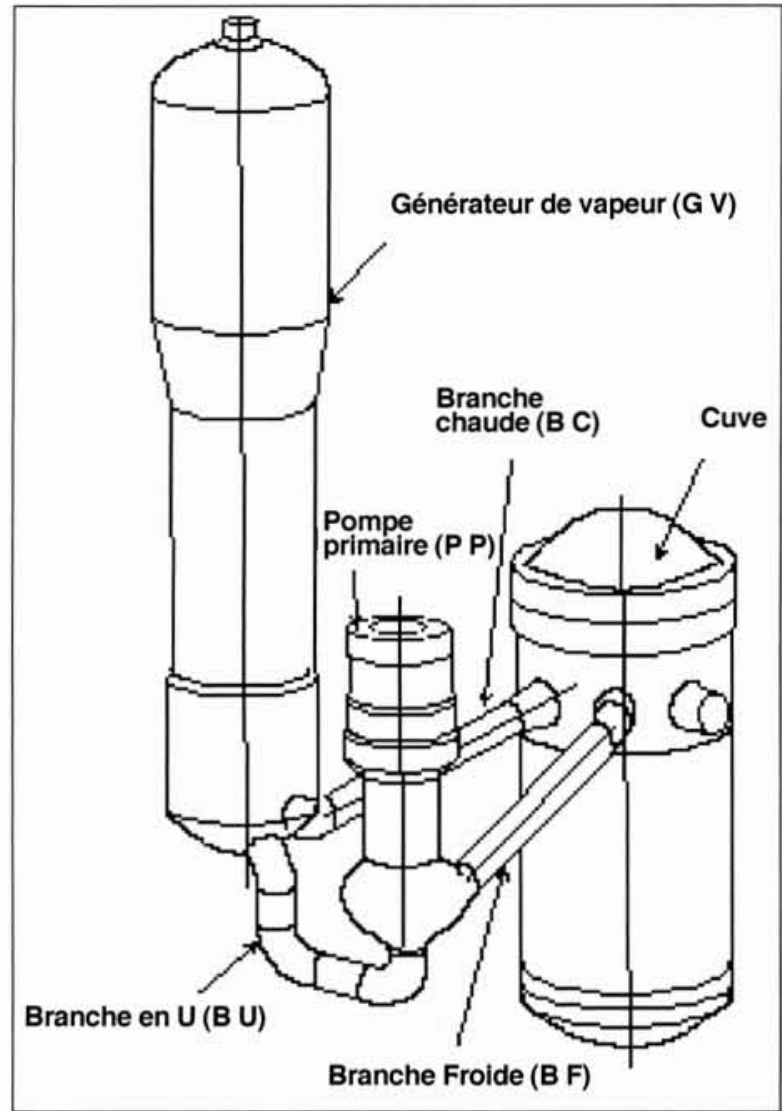

9. Vue d'une boucle du CPP.

Cette vérification passe par l'estimation des marges de dimensionnement du circuit primaire vis-à-vis du risque d'APRP. L'évaluation des marges de dimensionnement consiste, après avoir analysé les choix de modélisations et les hypothèses simplificatrices associés aux calculs de conception, à évaluer la différence (en termes d'efforts, de déplacements en des points choisis de la structure étudiée) entre une évaluation enveloppe issue de calculs de type dimensionnement et celles issues de calculs "best - estimate".

Pour mener à bien les calculs "best - estimate", on a développé et mis en œuvre des méthodes, modèles et outils, capables de représenter le comportement avec plus de réalisme qu'en dimensionnement. Ces progrès rendent possible la réalisation de nouveaux calculs, où la physique des phénomènes mis en jeu et les sources d'excitation peuvent être modélisées sans cesse plus finement. Les modèles mécaniques utilisés sont plus élaborés que par le passé, et le couplage entre les différentes phases de ces calculs est lui-même réalisé dans de meilleures conditions.

EDF a entrepris de fédérer les analyses correspondantes dans le cadre du projet CACIP "Comportement mécanique Accidentel du CIrcuit Primaire", piloté par la Division R\&D. La démarche proposée repose sur le chaînage de deux codes de calcul. Le code PLEXUS du CEA est qualifié dans le domaine de l'APRP. Il permet d'estimer le chargement hydrodynamique sur le circuit. La réponse mécanique du circuit peut ensuite être calculée à l'aide du Code_Aster ${ }^{\circledR}$, code de mécanique développé par la Division Recherche \& Développement d'EDF pour de nombreuses autres applications, comme l'ont montré les paragraphes précédents.
Les calculs s'effectuent en deux étapes. Premièrement, on calcule le champ transitoire d'efforts hydrodynamiques. Ces efforts sont liés, d'une part à la chute de pression interne à la brèche, et d'autre part à la propagation d'un front d'onde de décompression dans le circuit. Le calcul est réalisé en structures fixes. L'hypothèse des parois rigides est justifiée par la présence des dispositifs anti-fouettement limitant les déplacements des tuyauteries du CPP. Deuxièmement, le champ de pression calculé est appliqué au modèle mécanique du $\mathrm{CPP}$ pour calculer sa réponse dynamique et vérifier qu'il n'y a pas de perte d'intégrité dans d'autres composants du circuit primaire.

Pour calculer avec PLEXUS le chargement hydrodynamique dû à la rupture brusque du circuit, un modèle hydrodynamique filaire du fluide primaire a été élaboré. En supposant un écoulement monodirectionnel (hypothèse d'ondes planes), tous les volumes du CPP contenant le fluide primaire ont été modélisés par des "tuyaux équivalents" dont les longueurs et les diamètres respectent le parcours moyen du fluide primaire et les volumes fluide considérés. Le comportement du fluide primaire à l'intérieur du CPP est gouverné par les équations d'Euler avec un modèle du fluide diphasique de type homogène équilibré. Pour prendre en compte le caractère très rapide de la décompression du fluide à la brèche, un modèle diphasique plus précis est utilisé. Il s'agit du modèle non équilibré de Moody qui permet la prise en compte du glissement entre phases liquide et vapeur et la modélisation du débit critique diphasique au niveau de la brèche.

Avec ce modèle, le régime stationnaire dans un circuit sain est d'abord calculé, ce qui permet de s'assurer de la cohérence numérique du modèle PLEXUS vis-à-vis des données nominales issues du dossier de conception. Ensuite, une rupture brusque de la branche en " $U$ " en sortie du générateur de vapeur de l'une des boucles du CPP est simulée. La chute brutale de pression en une milliseconde jusqu'à la pression de saturation provoque l'apparition de deux ondes de décompression avec un front assez raide, qui se propagent dans le CPP de part et d'autre de la brèche. Le champ de pression transitoire calculé par PLEXUS est ensuite appliqué au modèle mécanique du CPP.

Dans le modèle mécanique élaboré avec le Code_Aster ${ }^{\otimes}$, les tuyauteries du CPP sont discrétisées à l'aide d'éléments finis de type "tuyaux" qui combinent une cinématique de poutre et une cinématique de coque. Ces éléments permettent de prendre en compte, non seulement les déformations de flexion et de torsion, mais aussi le gauchissement des sections, le gonflement et l'ovalisation des sections des tuyauteries du CPP dus à la pression. A cause d'une très forte pression interne (155 bars), ces dernières propriétés sont importantes pour la prédiction du comportement dynamique des tuyauteries qui peuvent plastifier localement dans les coudes. Des poutres de Timoshenko sont en revanche utilisées pour modéliser les composants du CPP plus rigides comme les générateurs de vapeur, les pompes et le corps du réacteur. Enfin, des éléments spéciaux dits de choc sont introduits dans le modèle mécanique pour modéliser les butées anti-fouettement. Pour minimiser le temps de calcul, les propriétés élasto-plastiques de matériaux ne sont prises en compte que dans les zones où les contraintes dépassent la limite élastique. Ces zones sont repérées à partir d'un calcul purement élastique.

En conclusion, l'ensemble de cette démarche permettra à EDF de monter en compétence dans le domaine du dimen- 
sionnement à l'APRP du circuit primaire. Les comparaisons des résultats issus des approches actuelles de dimensionnement et des nouvelles méthodes de résolution mises en oeuvre dans le cadre du projet CACIP permettront d'avoir une vision plus précise des marges actuelles de dimensionnement.

\section{VII ש LES PROGRÈS À ACCOMPLIR PAR L'INDUSTRIEL DEMEURENT NOM- BREUX}

Comme on a pu le constater, de nombreux problèmes de couplage fluide-structure peuvent se poser à une entreprise du secteur industriel, même lorsque le métier principal de cette dernière n'est pas la mécanique. Pour sa part, l'industriel EDF a fait le choix de développer les compétences nécessaires à la résolution de ces problèmes au sein d'une Division spécifique chargée de la R\&D, intégrée à l'entreprise. Ce choix a permis de favoriser les échanges entre les équipes en charge des différents problèmes. Il est apparu qu'une juste dose d'approche disciplinaire des différents problèmes posés peut apporter synergies et économies d'échelle. Dans la mesure où la maîtrise du couplage fluide-structure apparaît nécessaire dans bien des cas, cette discipline est jugée importante par la Division R\&D, ce qui a justifié la constitution d'un groupe de travail transverse à l'organisation principale de la Division.

Les cinq exemples d'application présentés dans cet article attestent du niveau de maîtrise des interactions fluide-structure d'ores et déjà atteint. Cependant, l'expérience de l'industriel montre aussi que de nombreux progrès sont encore devant nous. Ainsi, si les performances des algorithmes de calcul modal ne sont aujourd'hui plus à démontrer, la prévision du comportement modal des structures industrielles bute toujours sur le niveau de qualité des données fournies à ces mêmes algorithmes : méconnaissance relative des caractéristiques dynamiques des matériaux, simplification importante des conditions aux limites ... On note certes la convergence de plus en plus forte entre, d'une part la géométrie réelle des pièces, et d'autre part la représentation qui en est faite dans les codes de calcul à l'aide des méthodes de discrétisation. Un saut technologique est en cours, avec la banalisation croissante de la CAO et des méthodes de discrétisation "as built".

Dans le domaine du comportement du fluide, le coût des calculs demeure un frein, plus encore qu'en mécanique. La modélisation fine des écoulements diphasiques fait notamment encore l'objet de débats d'experts. Cependant, même les écoulements monophasiques, s'ils sont très turbulents comme le ruissellement d'un jet après impact sur l'auget mobile d'une roue PELTON, sont encore inabordables par simulation numérique.

Pour conclure, dans un contexte en constante évolution, les Unités Opérationnelles et la Division R\&D répondent de leur mieux aux problèmes posés à EDF, en s'appliquant à mettre en œuvre les progrès validés les plus récents.

\section{Remerciements}

Le Groupe Interactions Fluide-Structure remercie les départements MFTT, AMV et MTC de la Division R\&D ainsi que les personnes qui ont contribué à la rédaction du présent article, en particulier MM. Billet, Seligmann, Toulemonde, Scliffet, Archambeau, Hérard et Potapov.

\section{Bibliographie}

[1] "Calcul de la réponse dynamique de la roue PELTON de la Combe d'Avrieux". L. BILLET. ASTIER J.G. - , note interne de la Division R\&D HP-51/98/042/A.

[2] "CIRCUS un outil intégré pour les études de tuyauteries" D. SELIGMANN, I. FOURNIER, J. GUILLOU, V. VILLOUVIER, Revue EDF EPURE, $n^{\circ} 60$, Octobre 98, pp. 27-32

[3] "Etudes industrielles de vibrations sous écoulement dans des réseaux de tuyauteries". D. SELIGMANN, I. FOURNIER - La Houille Blanche n³/4 1998. pp. 134-139.

[4] "Simulation tridimensionnelle d'une soupape GV à l'aide du code N3SNATUR". L. LEAL DE SOUSA -, note interne de la Division R\&D HE-41/98/031/A

[5] "A time dependent scheme to compute flashing flows", Second International Symposium on Two Phase Flow Modelling and Experimentation. Pise (Italie), 23-26 mai 1999.

[6] "GEVIBUS, un outil-métier pour l'analyse prédictive des endommagements liés à l'interaction. fluide-structure dans les générateurs de vapeur" - N. GAY, C. BARATTE -, article présenté à la journée 98 du Club des Utilisateurs de Code_Aster

[7] "Comportement mécanique accidentel du circuit primaire" - Fe WAECKEL. J. BEDIOU - Article présenté à la journée 99 du Club des Utilisateurs de Code_Asterill.

[8] "PLEXUS. A general computer program for the fast dynamic analysis. The case of pipe-circuits", M.LEPAREUX, B.SCHWAB, H.BUNG, Proc. Smirt 8, Vol F1 2/1, 1985.

[9] Plaquette de présentation du Code_Aster" : Analyse des Structures et Thermo-mécanique pour des Etudes et des Recherches, 1998, HI$75 / 98 / 020$.

[10] "Le Code_Aster" : un produit pour les mécaniciens des structures" J. R. LEVESQUE, Revue EDF EPURE, $n^{\circ} 60$, octobre 98, pp. 7-20. 